Yuefang Zhang*, Shunhua Liu and wanJun Hao

\title{
Microwave absorption and mechanical properties of double-layer cement-based composites containing different replacement levels of fly ash
}

DOI 10.1515/secm-2016-0325

Received November 1, 2016; accepted February 11, 2017; previously published online May 11, 2017

Abstract: Double-layer absorbing cement-based composites with the thickness of $10 \mathrm{~mm}$ were prepared, including different replacement levels of fly ash (FA) in the absorbing layer as well as the matching layer for impedance matching. Waste polyethylene terephthalate bottle fragment was introduced as electromagnetic transparent reinforcement aggregate. Carbon black was used to be original absorbent in the absorbing layer. The microstructure and electromagnetic parameters of FA were closely looked at through scanning electron microscope, X-ray diffraction, and analyzer of vector network. The absorption and mechanical properties of cement-based composites were tested. It turned out that when the optimal replacement ratio of FA in the absorbing layer and matching layer gets to 50\%:30\%, the minimum value of reflection loss achieves $-22.3 \mathrm{~dB}$ at 13.2 $\mathrm{GHz}$; also, the value of absorption bandwidth that is effective $(<-8 \mathrm{~dB})$ is $6.4 \mathrm{GHz}$. Ni-Zn ferrite proves to be a feasible absorbent that is additional for the matching layer compared to what is added to the absorbing layer. The compressive strength of all the mixtures decreased, while the flexural strength decreased first and then increased with the rise of the FA replacement level.

Keywords: absorption properties; cement composites; FA; mechanical properties.

\section{Introduction}

The extensive development of communication technology and electronic systems led to health concerns about

\footnotetext{
*Corresponding author: Yuefang Zhang, School of Materials and Engineering, Dalian University of Technology, Dalian, 116085, China; and School of Materials Science and Chemical Engineering, Hainan University Haikou, 570228, China, e-mail: yuefang3900@163.com Shunhua Liu: School of Materials and Engineering, Dalian University of Technology, Dalian, 116085, China

wanJun Hao: School of Materials Science and Chemical Engineering, Hainan University Haikou, 570228, China
}

pollution brought about by electromagnetic waves. Therefore, electromagnetic interference prevention for buildings is growingly relevant under current circumstances. Cement-based electromagnetic absorbing composites that boast sound environmental adaptability, low cost, and rich resources have become the focus of studies on materials [1].

Cement is helpful to some extent with its low electromagnetic absorption capacities. The addition of fillings and absorbents, such as carbon [2,3], ferrites [4], and fibers $[5,6]$ is a feasible way to heighten the absorption of material that is cement-based. Moreover, the filling of electromagnetic transparent beads like expanded perlite and expanded polystyrene (EPS) has been proposed to enhance the impedance matching characteristic between free space and the surfaces of cement composites [7, 8].

Fly ash (FA), which is a type of by-product of coalburning thermal power stations, has often been incorporated in structural and paving concrete mixes as a supplementary cementitious material to reduce $\mathrm{CO}_{2}$ emissions resulting from cement and concrete industry. There are three aspects of the main effects of FA on concrete: pozzolanic effect, morphologic effect, and microaggregate effect, all of which contribute to the durability and excellent mechanical properties of concrete [9]. In addition, $\mathrm{SiO}_{2}$ and $\mathrm{Al}_{2} \mathrm{O}_{3}$ constitute the main components of FA, which are both electromagnetically transparent. As a result, some works have been done to examine the possibility that FA can be a cement replacement to modify the characteristic impedance of cement materials, which improves shielding/absorbing properties [10-12]. Li et al. [13] improved the absorbing properties by introducing low-quality FA and EPS in cement composites.

Relevant literatures [14, 15] show that double-layer absorbers, which boast a greater number of controllable parameters for adjusting the absorbing strength and impedance matching condition, possess absorption bandwidth that is wider and reflection loss (RL) that is larger compared to the single-layer absorber in $\mathrm{GHz}$ frequency. Zhang et al. [16] prepared single-layer as well as doublelayer cement absorbers whose thickness is $30 \mathrm{~mm}$ by means of Mn-Zn ferrite absorbents. Compared to a singlelayer structure, double-layer cementitious plates work 
to reduce the reflectivity by an average of $6-8 \mathrm{~dB}$, with a maximum reduction of $-15 \mathrm{~dB}$ obtained with plates prepared with the surface layer of $30 \mathrm{wt} . \%$ silica fume mortar as well as the loss layer of $30 \mathrm{wt} . \% \mathrm{Mn}-\mathrm{Zn}$ ferrite mortar.

In this research, a new type of double-layer microwave absorbing cement composite reinforced with waste polyethylene terephthalate (PET) fragment and FA in both absorbing and matching layers was designed and fabricated. Carbon black (CB) and Ni-Zn ferrite were employed to be the original absorbents in their corresponding layer according to corresponding conductive and magnetic characters. Also, FA powder was characterized in a systemic manner, including X-ray diffraction (XRD), scanning electron microscope (SEM), and electromagnetic performances. By looking at influences of FA in every layer, the ideal replacement ratio of FA in both the matching layer and the absorbing layer could be determined.

\section{Materials and experiment}

\subsection{Materials}

According to Chinese Standard GB175-2007, Ordinary Portland Cement supplied by Hainan Tianya Cement Corporation in China was used. FA was obtained from Shanghai Baogang power plant. According to GB/T 1596-2005 standard, it was classified as grade II. Table 1 presents the chemical compositions of FA and cement. The size of PET fragment was 3-8 mm and it had an irregular shape. Ni-Zn ferrite (Ni0.5Zn0.5Fe2O4) was analytically pure and purchased from Shenzhen HC Magnetic Materials Co., LTD. N234 CB was milled in a ball milling for a duration of $3 \mathrm{~h}$ before it was used.

\subsection{Sample preparation}

The cement-based plates used for the RL measurement were prepared as follows. To prepare the absorbing layer, first, the cement and PET fragment were spread and dry mixed for 2 min by a mortar mixer. Second, FA, absorbent powder, and water were put into it and then mixed for $10 \mathrm{~min}$ with a water/cement ratio of 0.35 . Finally, the mixture of cement was poured into the oiled molds whose size is $200 \times 200 \times 5 \mathrm{~mm}^{3}$, vibrated simultaneously to remove any air entrapped and was smoothed by virtue of a float. When the initial setting of the layer at the bottom was done, the matching layer which was as thick as the bottom layer was added. After a day, the samples were firstly demolded and then cured in water for 28 days at $25 \pm 2^{\circ} \mathrm{C}$. The arched testing method was applied to measure the RL by virtue of a HP8720B vector network analyzer in an anechoic chamber. The test frequency ranges from 2 to $18 \mathrm{GHz}$. The mixed proportions of the samples are presented in Table 2 .

The samples used for electromagnetic parameter measurement were prepared by mixing 50 vol.\% FA

Table 1: Cement and fly ash chemical compositions (mass\%).

\begin{tabular}{lrrrrrrrrr}
\hline & $\mathrm{SiO}_{2}$ & $\mathrm{Al}_{2} \mathbf{O}_{3}$ & $\mathrm{Fe}_{2} \mathbf{O}_{3}$ & $\mathrm{CaO}$ & $\mathrm{MgO}$ & $\mathrm{SO}_{3}$ & $\mathrm{~K}_{2} \mathbf{O}$ & $\mathrm{Na}_{2} \mathbf{O}$ & Loss on ignition \\
\hline Cement & 20.13 & 5.62 & 4.29 & 62.08 & 2.84 & 2.31 & 0.87 & 0.21 & 3.60 \\
Fly ash & 50.22 & 31.77 & 5.53 & 3.49 & 1.07 & 0.65 & 1.38 & 0.65 & 1.45 \\
\hline
\end{tabular}

Table 2: The mixed proportion of double-layer cement composites.

\begin{tabular}{|c|c|c|c|c|c|c|c|c|c|c|c|}
\hline \multirow[t]{2}{*}{ Sample } & \multicolumn{5}{|c|}{ Matching layer (vol\%) } & \multicolumn{6}{|c|}{ Absorbing layer (vol\%) } \\
\hline & Cement & FA & PET & Ferrite & Thickness (mm) & Cement & FA & PET & CB & Ferrite & Thickness (mm) \\
\hline 1 & 100 & - & 5 & - & 5 & 100 & - & 3 & 4 & - & 5 \\
\hline 2 & 80 & 20 & 5 & - & 5 & 80 & 20 & 3 & 4 & - & 5 \\
\hline 3 & 60 & 40 & 5 & - & 5 & 80 & 20 & 3 & 4 & - & 5 \\
\hline 4 & 40 & 60 & 5 & - & 5 & 80 & 20 & 3 & 4 & - & 5 \\
\hline 5 & 50 & 50 & 5 & - & 5 & 90 & 10 & 3 & 4 & - & 5 \\
\hline 6 & 50 & 50 & 5 & - & 5 & 80 & 20 & 3 & 4 & - & 5 \\
\hline 7 & 50 & 50 & 5 & - & 5 & 70 & 30 & 3 & 4 & - & 5 \\
\hline 8 & 50 & 50 & 5 & - & 5 & 90 & 10 & 3 & 4 & 4 & 5 \\
\hline 9 & 50 & 50 & 5 & 4 & 5 & 90 & 10 & 3 & 4 & - & 5 \\
\hline
\end{tabular}


power and 50 vol.\% paraffin and were later compressed into toroid-shaped specimens whose inner diameter was $3 \mathrm{~mm}$, outer diameter was $7.0 \mathrm{~mm}$, and thickness was $2 \mathrm{~mm}$. The electromagnetic parameters of FA were examined by virtue of the coaxial flange way with a HP8720B analyzer of vector network whose range of test frequency was $2-18 \mathrm{GHz}$. The microstructures and the mineralogical composition of FA were examined by XRD and SEM.

The size of specimens for the mechanical properties were $40 \times 40 \times 40 \mathrm{~mm}^{3}$ and $160 \times 40 \times 40 \mathrm{~mm}^{3}$, respectively. The compressive and flexural strength of specimens at the curing periods of 7 days and 28 days were tested according to GB/T17671-1999. Three samples were tested for each mix. and the average value was used as the final compressive and flexural strength.

\section{Results and discussion}

\subsection{Features of FA powder}

The XRD spectra of FA are illustrated in Figure 1. A series of sharp XRD peaks were found in the crystalline planes, which are identified as mullite, quartz, and minor quantities of hematite and lime. Additionally, a pronounced broad hump of amorphous vitreous materials can be observed in the background between $16^{\circ}$ and $35^{\circ} 2 \theta$.

The morphology and chemical compositions of FA particles are controlled mainly by the source of coal, the combustion temperature, and cooling rate. As shown in Figure 2, according to SEM photograph, FA samples comprise irregular unburned carbon, cenospheres, and solid spheres. The present study has also observed mineral aggregates containing corundum, quartz, and magnetite particles [17]. $\mathrm{SiO}_{2}$ and $\mathrm{Al}_{2} \mathrm{O}_{3}$ are rich in most particles, which are electromagnetically transparent and contribute about $82 \%$ of weight. So FA with the cenosphere structure serves to offer sufficient transmission paths to incident wave, which could be employed to modulate the impedance matching of cement composites.

\subsection{The electromagnetic properties of FA}

The storage capacity of magnetic and electric energy is embodied in real parts of complex permeability and permittivity, and the loss of energy is symbolized by the imaginary parts. In this paper, in the absorbing layer CB is employed as absorbent because of its large permittivity and excellent dielectric loss character. $\mathrm{Ni}-\mathrm{Zn}$ ferrite is

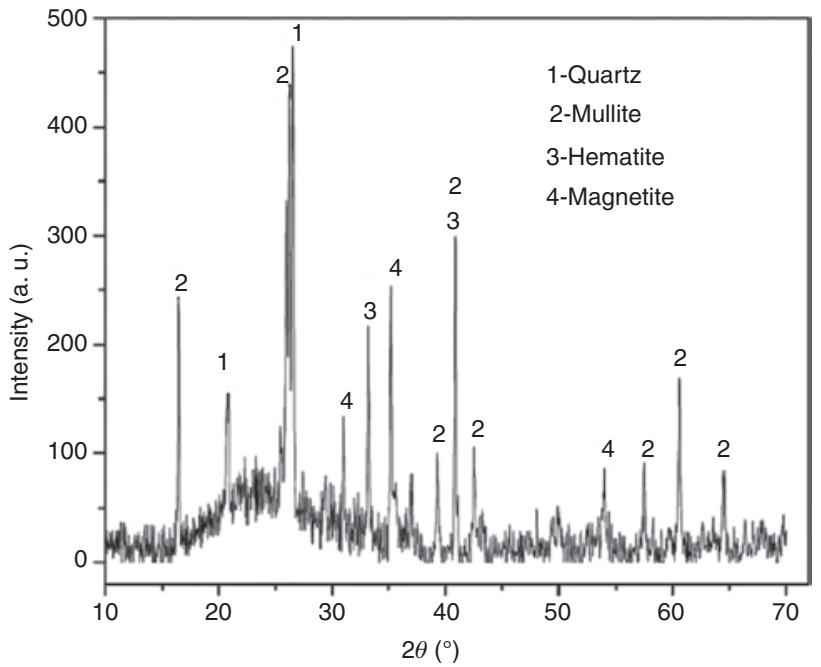

Figure 1: XRD pattern of fly ash.

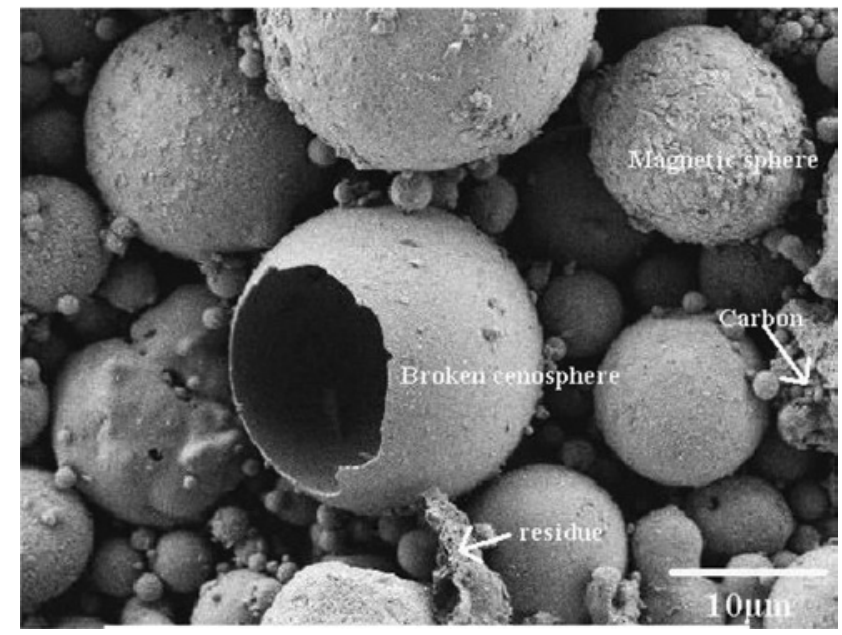

Figure 2: SEM image of fly ash.

used to be magnetic fillers in the absorbing layer or the matching layer. The electromagnetic characteristics of $\mathrm{CB}$ and Ni-Zn have been explained elsewhere [18]. Here the complex permeability and permittivity of FA have been studied specifically, as shown in Figure 3A and B. The real parts of complex permeability and permittivity have been almost constant, which are 1.01 and 3.15 within the range of 2-18 GHz. Also, the imaginary parts of permeability and permittivity are 0.01 and 0.07 , which means that the microwave absorption property of FA is largely due to the dielectric loss instead of magnetic loss. Its dielectric loss is mainly ascribed to the conduction of unburned carbon, dielectric relaxations of metal oxides, and the complicated interaction of defects and particles with incident wave in FA [13]. Furthermore, the main components of $\mathrm{SiO}_{2}$ and 

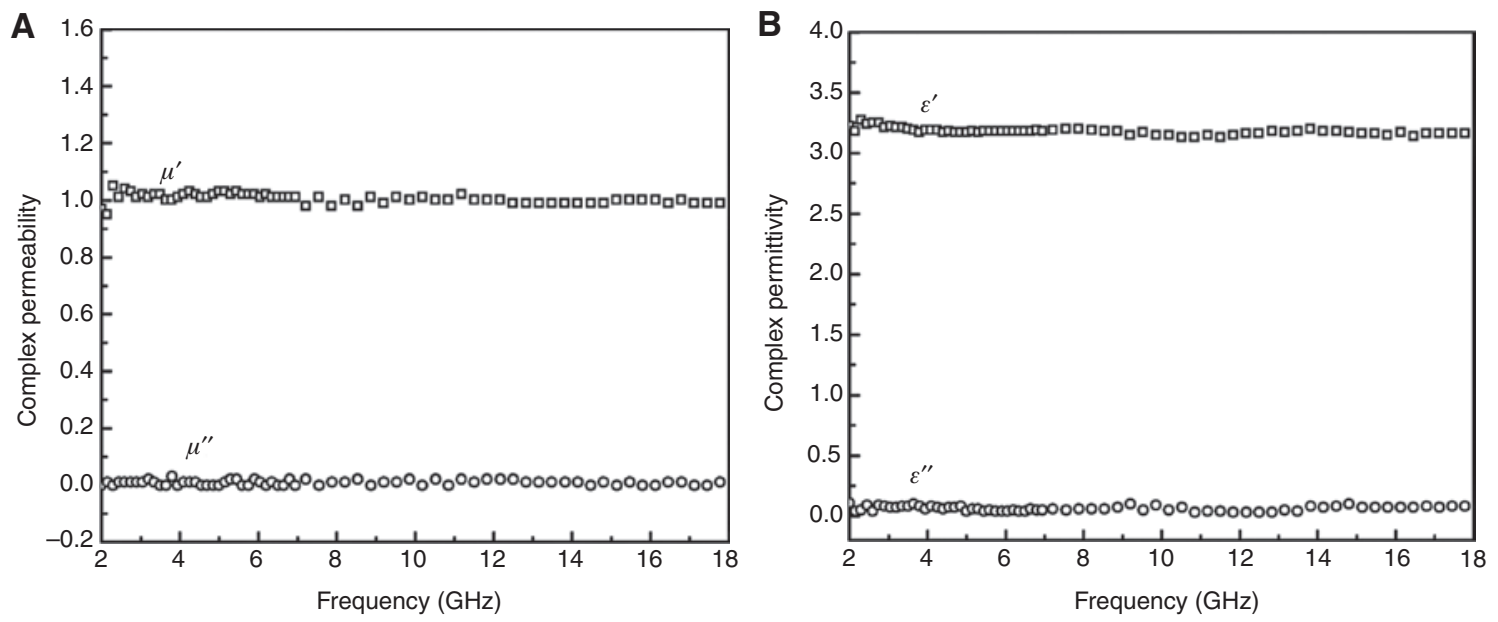

Figure 3: Complex permeability (A) and complex permittivity (B) of FA in 2-18 GHz range.

$\mathrm{Al}_{2} \mathrm{O}_{3}$ with special porous structure in FA can ameliorate the impendence matching between the atmosphere and the double layers of cement composite.

\subsection{Electromagnetic wave absorbing property}

\subsubsection{Theory}

\subsubsection{The impedance characteristics of double-layer cement composites}

On the basis of the electromagnetic wave transmission line theory, when it comes to a double-layer absorber comprising the matching layer as the first layer as well as the absorption layer as the second layer, as shown in Figure 4, the input impedance $Z_{\text {in }}$ of the absorber backed by a perfect conductor is given by Equation (1) [19, 20]:

$$
Z_{\text {in }}=Z_{1} \frac{Z_{1} \tanh \left(k_{1} d_{1}\right)+Z_{2} \tanh \left(k_{2} d_{2}\right)}{Z_{1}+Z_{2} \tanh \left(k_{1} d_{1}\right) \tanh \left(k_{2} d_{2}\right)}
$$

where $Z_{i}=\sqrt{\mu_{0} \mu_{i} / \varepsilon_{0} \varepsilon_{i}}, k_{i}=\omega \sqrt{\varepsilon_{i} \varepsilon_{0} \mu_{i} \mu_{0}}$

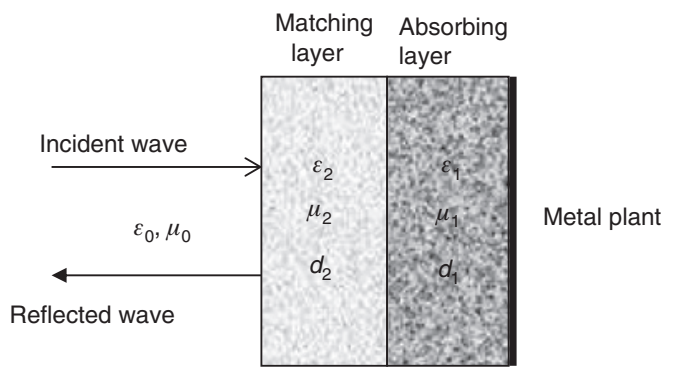

Figure 4: Schematic illustration of the double-layer cement composite consisting of a matching layer and an absorbing layer. $d_{1}, Z_{1}$, and $k_{1}$ in the matching layer, as well as $d_{2}, Z_{2}$, and $k_{2}$ in the absorption layer, are the layer thickness, propagation constant, and characteristic impedance, respectively. $Z_{\text {in }}$ is the input impedance; $\varepsilon_{0}$ and $\mu_{0}$ are the permittivity and permeability of vacuum. The propagation constant and characteristic impedance are expressed by the complex permeability $\mu\left(\mu=\mu^{\prime}-j \mu^{\prime \prime}\right)$ and the complex permittivity $\varepsilon\left(\varepsilon=\varepsilon^{\prime}-\varepsilon^{\prime \prime}\right)$. The RL is shown below $[19,20]$ :

$$
\mathrm{RL}=201 \mathrm{~g}\left|\frac{Z_{\text {in }}-Z_{0}}{Z_{\text {in }}+Z_{0}}\right|(\mathrm{dB})
$$

where $Z_{0}$ is the feature impedance of a vacuum, $Z_{0}=\sqrt{\mu_{0} / \varepsilon_{0}}=120 \pi \Omega$. As is deduced from Equations

and (2), the absorption characteristic of the absorber with double layer is improved effectively when multiple parameters are modulated, including complex permeability $\mu$, complex permittivity $\varepsilon$, and the thickness $d$ of the absorbing layer and the matching layer to make $Z_{\text {in }}$ more proximate to $Z_{0}$.

\subsubsection{Scattering and absorption of FA and PET}

On the one hand, PET is a transparent material to electromagnetic wave, and there are many porous particles in FA considered to be electromagnetically transparent too. Cement is uniformly coated on PET and porous particles, which formed a core-shell structure as shown in Figure 5A and $\mathrm{B}$, of which the cores are the cavities of PET fragment and porous particles in FA. Also, the shells are actually cement walls, as is demonstrated in Figure $5 \mathrm{~A}$ and B. The incident wave could be ameliorated by the scattering, dielectric losses, and complex refraction by cement matrix when propagating in the specimen. 
A
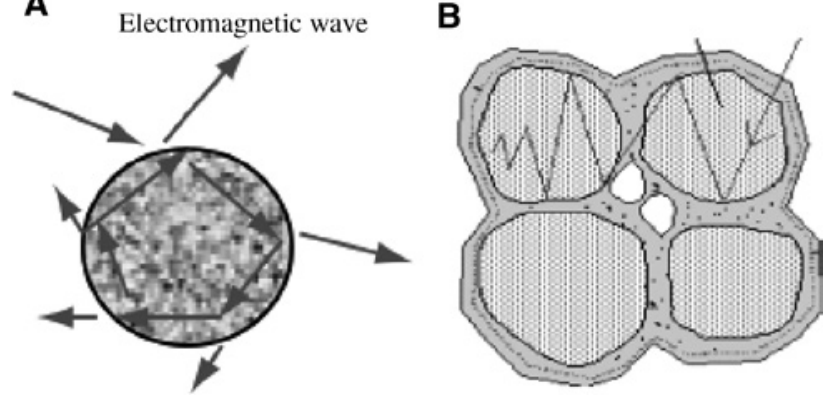

Figure 5: Reflection and scattering of the hollow spheres in FA on electromagnetic wave. (A) Single hollow sphere. (B) Multiple hollow sphere.

Figure $5 \mathrm{~A}$ and $\mathrm{B}$ show the phenomenon of reflection and multiple scattering of electromagnetic wave in single cavity and multiple granules. When electromagnetic wave makes its entry to the absorber along $x$-axis direction, the interface between air and absorber is $x=0$; the energy of scattering wave at $x$ point $I(x)$ is shown below [21]:

$$
I(x)=I_{0} \exp \left(-n \frac{k^{4}\left(\left|\varepsilon_{r}-1\right|^{2}+\left|\mu_{r}-1\right|^{2}\right)}{6 \pi} \cdot x\right)
$$

where $I_{0}$ is the energy of incident, $n$ is the number of hollow beads in one unit volume, $k=\omega \sqrt{\varepsilon_{0} \varepsilon_{r} \mu_{0} \mu_{r}}$ is wave number in composite basis, and $\mu_{r}$ and $\varepsilon_{r}$ are the permeability and permittivity of cement composites. It can be seen from the formula that the scattering attenuation is in close correlation with the ratio of porous beads, the permittivity and the permeability. Also, the multiple scattering among cavity increases the attenuation times in single hollow cavity. As a result, through the introduction of absorbents in the cement walls as well as the rise of hollow cavity content, more energy is ameliorated by enhancing the attenuation probabilities.

\subsubsection{The influence of FA in matching layer on absorption performance}

Table 2 demonstrates the design of absorbing layer and matching layer of samples $1 \# \sim 9 \#$, in which the thickness of the matching layer as well as the absorption layer are the same, $5 \mathrm{~mm}$. The RL of double-layer samples with different levels of FA replacement cement in matching layer is demonstrated in Figure 6, and relevant data are shown in Table 3. From Figure 6 and Table 3, we can see that the rise of FA content improves the microwave absorption. The optimal value of RL achieves $-15.4 \mathrm{~dB}$, and the effective absorption band (less than $-8 \mathrm{~dB}$ ) arrives at 5.9

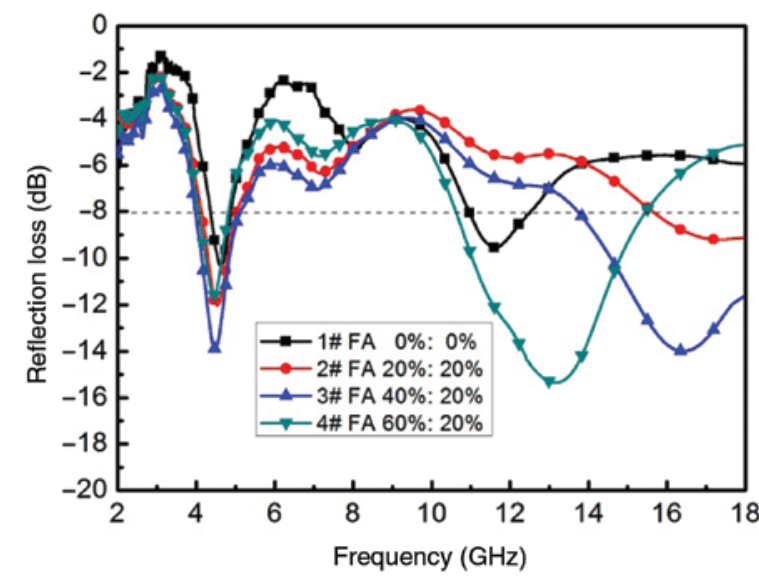

Figure 6: Reflection loss of double-layer cement samples with different replacement level of FA in matching layer.

Table 3: Detailed data of double-layer cement composites with different replacement levels of FA in the matching layer.

\begin{tabular}{lrrr}
\hline Sample & $\begin{array}{r}\text { Optimal } \\
\text { RL (dB) }\end{array}$ & $\begin{array}{r}\text { Absorption band for } \\
\mathbf{R L}<-8 \mathbf{d B} / \mathbf{G H z}\end{array}$ & $\begin{array}{r}\text { Bandwidth for } \\
\mathbf{R L}<-8 \mathrm{~dB} / \mathbf{G H z}\end{array}$ \\
\hline 1 & -10.3 & $4.3-4.9,10.9-12.5$ & 2.2 \\
2 & -12.1 & $4.1-5.0,15.6-18.0$ & 3.3 \\
3 & -14.0 & $3.9-5.1,13.7-18$ & 5.7 \\
4 & -15.4 & $4.0-4.9,10.5-15.5$ & 5.9 \\
\hline
\end{tabular}

$\mathrm{GHz}$, when the volume fraction of FA replacement cement reaches $60 \%$. By contrast, for the FA-free cement plate the minimum RL is $-10.3 \mathrm{~dB}$ and the effective asorption bandwidth (better than $-8 \mathrm{~dB}$ ) is only $2.2 \mathrm{GHz}$.

Apparently, the additional FA is conducive to lowering the peak value and expanding the absorbing bandwidth. Compared with free space, cement has higher permittivity and compact structure that leads to impedance mismatch and limits incidental wave transmission. Therefore, owing to the low complex permittivity, FA as well as PET are introduced to modulate the impedance matching feature of the cementitious composites. At the same time, the space network structures consisting of PET fragments and the cenospheres in FA bring about channels for the transmission of electromagnetic wave. In conclusion, the FA in the matching layer is of vital importance to reduce the peak values and expand the absorption bandwidth.

\subsubsection{The influence of FA in absorbing layer on absorption performance}

Figure 7 and Table 4 exhibit the RL curve and the corresponding data in detail of samples $5 \#, 6 \#$, and $7 \#$ with the 


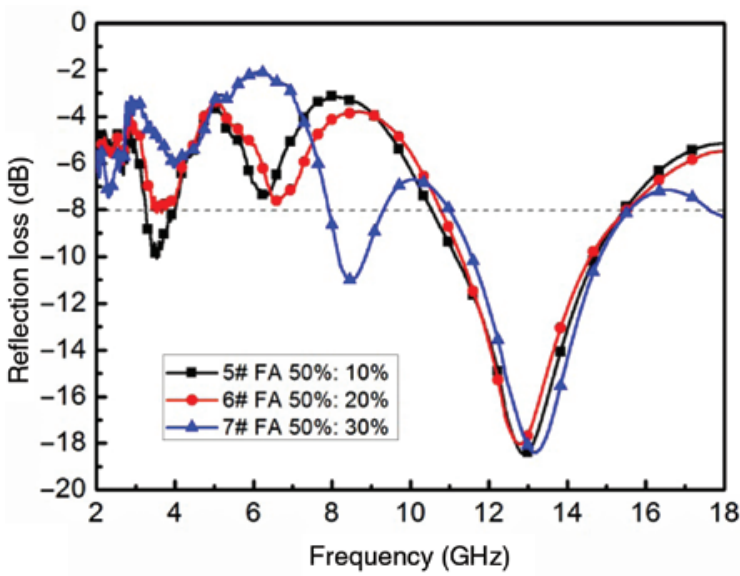

Figure 7: Reflection loss of double-layer cement samples with different replacement level of FA in absorbing layer.

same matching layers of 50\% FA and 5\% PET. The absorbing layers are filled with 4\% CB, 3\% PET, and 10\% (5\#), $20 \%$ (6\#), and 30\% (7\#) FA, respectively.

It can be seen that as the FA content (varied from $10 \%, 20 \%$, to $30 \%$ ) in the absorbing layers increases, the peak positions (about $13 \mathrm{GHz}$ ), peak value $(-18.4,-18.1$, and $-18.4 \mathrm{~dB}$ ), and the absorption band for $\mathrm{RL}<-8 \mathrm{~dB}$ (11.0-15.6, 10.8-15.5, and $10.5-15.5 \mathrm{GHz})$ are found to exhibit no obvious changes in the high-frequency region, while the value of low-frequency absorption peaks changes and the peak positions get closer to the high-frequency region. It is due to the fact that increasing FA content gives rise to a low permittivity and increasing matching frequency, of which the scale-dependent effective medium theory is a proof [22]. The improved absorption properties can be ascribed to the matched characteristics impedance of the absorbing layer. Otherwise, the incident wave would be reflected partly on the interface of the absorbing layer as well as the matching layer. The effective absorption bandwidth will worsen as a result of the strong reflection, like sample 6\#. So there would be an optimal ratio of FA in the absorbing layer and the matching layer to obtain minimum RL value, which reaches $-22.3 \mathrm{~dB}$ at $13.2 \mathrm{GHz}$. Also, the effective absorption bandwidth $(<-8 \mathrm{~dB})$ arrives at $6.4 \mathrm{GHz}$ at the FA ratio of $50 \%: 30 \%$.

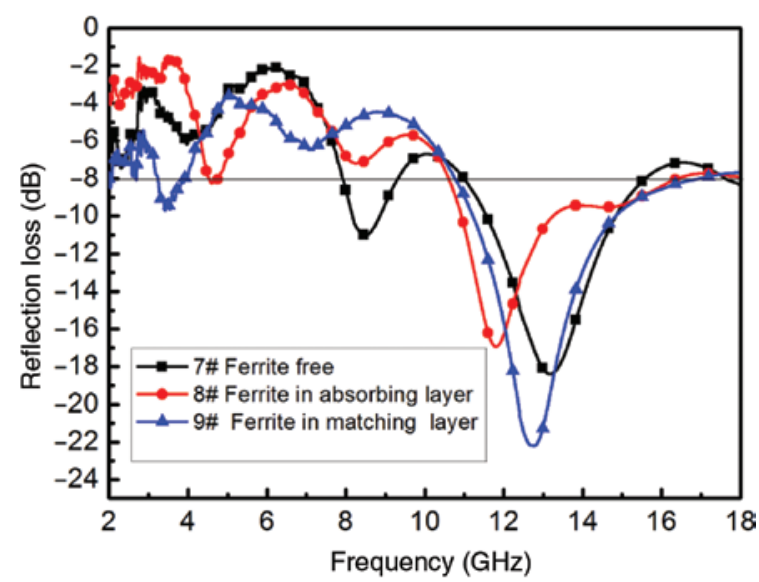

Figure 8: Effect of Ni-Zn ferrite on reflection loss of double-layer cement samples.

\subsubsection{The influence of Ni-Zn ferrite on absorption performance}

Figure 8 shows the influence on the absorbing performance of Ni-Zn ferrite in the absorbing layer (8\#) and the matching layer (9\#), and Table 5 presents the detailed relevant data. The optimal RL is $-22.2 \mathrm{~dB}$. Also, the bandwidth $(\mathrm{RL}<-8 \mathrm{~dB})$ is $7.2 \mathrm{GHz}$ for 9\#. Nonetheless, the value of effective absorption bandwidth $(\mathrm{RL}<-8 \mathrm{~dB})$ is $6.1 \mathrm{GHz}$, which peaks at $-17.0 \mathrm{~dB}$ for $8 \#$. The comparisons indicate that in the matching layer Ni-Zn ferrite can palpably improve absorption in the peak value as well as the bandwidth, but Ni-Zn ferrite in the absorbing layer serves to decrease absorbing properties compared to the Ni- $\mathrm{Zn}$ ferrite free sample (7\#). Ni-Zn ferrite is considered as a promising magnetic loss absorbent, which boasts high $\varepsilon$ and $\mu$. In the present study, an increase was observed in the composite ameliorating performance when $\mathrm{Ni}-\mathrm{Zn}$ ferrite was filled in the matching layer. The reflections of incident wave are also suppressed by the closer $\varepsilon$ and $\mu$. As a result, the peak value and the bandwidth are greatly improved. However, the introduction of $\mathrm{Ni}-\mathrm{Zn}$ ferrite in the absorbing layer has negative influence on absorption, which is possibly ascribed to the mismatching impedance of the matching layer and the absorption layer.

Table 4: Detailed data of double-layer cement composites with different replacement levels of FA in the absorbing layer.

\begin{tabular}{lrrr}
\hline Sample & Optimal RL/dB & Absorption band for $\mathbf{R L}<-\mathbf{8} \mathbf{~ d B} / \mathbf{G H z}$ & Bandwidth for RL $<-\mathbf{8 ~ d B} / \mathbf{G H z}$ \\
\hline 5 & -18.4 & $3.2-4.0,10.5-15.5$ & 5.8 \\
6 & -18.1 & $3.5-3.6,10.8-15.5$ & 4.8 \\
7 & -18.4 & $7.9-9.3,11.0-15.6,17.6-18$ & 6.4 \\
\hline
\end{tabular}


Table 5: Detailed data of double-layer cement composites with $\mathrm{Ni}-\mathrm{Zn}$ ferrite in different layers.

\begin{tabular}{lrrr}
\hline Sample & Optimal RL/dB & Absorption band for RL $<-8 \mathrm{~dB} / \mathrm{GHz}$ & Bandwidth for RL<-8 dB/GHz \\
\hline 7 & -18.4 & $7.9-9.3,11.0-15.6,17.6-18$ & 6.4 \\
8 & -17.0 & $4.5-4.8,10.6-16.4$ & 6.1 \\
9 & -22.2 & $3.2-4.0,10.7-17.1$ & 7.2 \\
\hline
\end{tabular}

\subsection{Effects on mechanical performance of the filling proportion of FA}

Table 6 exhibits the compressive strength of samples consisting of various replacement levels of FA ranging from 20.52 MPa to $35.44 \mathrm{MPa}$ on 7 days and ranging from $31.40 \mathrm{MPa}$ to $47.24 \mathrm{MPa}$ on 28 days. The compressive strength of mixtures showed a reduction that goes hand in hand with the rise of FA replacement level. The FA, CB-blended samples had a lower compressive strength than the cementitious mixtures of only FA replacement. The reduction in the compressive strength of the cementitious mixer was stopped by a prolonged curing period. For example, when the curing period lasted 7 days, it turned out that the compressive strength of the mixer with $60 \%$ FA was reduced by $42.01 \%$ in comparison to the FA free cement. However, in the same samples when the curing period lasted 28 days, the compressive strength was found to be down by $23.26 \%$. This could be because at the early curing period, the pozzolanic reaction activity of FA was low, and during the later stages of the curing period, the reaction of calcium hydroxide $(\mathrm{CH})$ as a result of the primary cement hydration and FA generated a secondary hydration $[9,23,24]$.

Table 6: Compressive strength and flexural strength of samples containing different replacement levels of FA.

\begin{tabular}{lrrrrr}
\hline Mixture no. & \multicolumn{2}{r}{$\begin{array}{r}\text { Compressive } \\
\text { strength/MPa }\end{array}$} & & \multicolumn{2}{r}{$\begin{array}{r}\text { Flexural strength/ } \\
\text { MPa }\end{array}$} \\
\cline { 2 - 3 } \cline { 5 - 6 } & $\mathbf{7}$ days & 28 days & & 7 days & 28 days \\
\hline FA00 & 35.44 & 47.24 & 7.22 & 8.64 \\
FA10 & 31.51 & 46.05 & 6.65 & 8.83 \\
FA20 & 29.83 & 41.85 & 7.31 & 9.12 \\
FA30 & 25.12 & 43.11 & 6.17 & 8.87 \\
FA40 & 24.24 & 37.62 & 5.94 & 8.48 \\
FA50 & 27.89 & 39.92 & 5.80 & 8.16 \\
FA60 & 20.52 & 34.25 & 5.54 & 7.50 \\
CB4FA00 & 33.16 & 42.71 & 6.11 & 6.68 \\
CB4FA10 & 27.16 & 39.86 & 5.48 & 5.70 \\
CB4FA20 & 22.20 & 35.46 & 5.17 & 6.87 \\
CB4FA30 & 25.77 & 31.40 & 5.87 & 6.40 \\
\hline
\end{tabular}

The flexural strength of samples decreases first and then rises with the replacement of cement by FA at the curing ages of 7 days and 28 days. The 20\% FA replacement cement exhibits the highest flexural strength (7.31 MPa at 7 days and $9.12 \mathrm{MPa}$ at 28 days) compared to others. For 4\% CB blended mortars, both flexural and compressive strength are reduced to a lower value compared to samples without.

\section{Conclusions}

In the present study, double-layer cement composites with the thickness of $10 \mathrm{~mm}$ by FA replacing cement partially in the absorbing layer and the matching layer were designed. The matching impendence between the free space and the two layers can be improved, which greatly influences the absorbing performance. When the optimal replacement ratio of $\mathrm{FA}$ in the absorbing layer as well as the matching layer reaches 30\%:50\%, the minimum value of RL achieves $-22.3 \mathrm{~dB}$ at $13.2 \mathrm{GHz}$, also, the effective absorption bandwidth $(<-8 \mathrm{~dB})$ reaches $6.4 \mathrm{GHz}$. Compared to what is added to the matching layer, Ni-Zn ferrite serves as a feasible additional absorbent for the matching layer. For the composite consisting of 50\% FA, 5\% PET, and 6\% Ni-Zn ferrite in the matching layer and 30\% FA, 3\% PET, and 4\% $\mathrm{CB}$ in the absorbing layer, the bandwidth below $8 \mathrm{~dB}$ is expanded to $7.2 \mathrm{GHz}$, and the optimal RL is $-22.2 \mathrm{~dB}$ in the range of frequency of 2-18 GHz. Compressive strength is reduced by the rise of content of FA, while flexural strength of samples decreases first and then increases. The extra addition of $\mathrm{CB}$ to the cementitious mixes is not conducive to compressive and flexural strength.

Acknowledgments: The authors acknowledge the support provided by Program for the National Natural Science Foundation of China (No. 51577021), the major projects of Hainan Province (No. ZDZX2013015), the Natural Science Foundation of Hainan province (No. 20165192), the Fundamental Research Funds Dalian University of Technology (No. DUT14YQ201) and the New Century Talent Support Program (No. NCET-13-0071). 


\section{References}

[1] Guan H, Liu S, Duan Y. Cement Concrete Comp. 2006, 28, 468-474.

[2] Dai Y, Sun M, Liu C, Li Z. Cement Concrete Comp. 2010, 32, 508-513.

[3] Cao J, Chung DDL. Carbon 2003, 41, 2433-2436.

[4] Oikonomou A, Giannakopoulou T, Litsardakis G. J. Magn. Magn. Mater. 2007, 316, 827-830.

[5] Chung DDL. Compos. Part B Eng. 2000, 31, 511-526.

[6] Atis CD, Karahan O. Constr. Build. Mater. 2009, 23, 392-399.

[7] Guan HT, Liu SH, Duan YP, Zhao YB. Cement Concrete Comp. 2007, 29, 49-54.

[8] Li B, Duan Y, Zhang Y, Liu S. Mater. Design 2011, 32, 3017-3020.

[9] Yamamoto T, Kanazu T, Nambu M. Fuel 2006, 85, 2345-2351.

[10] Cao J, Chung DDL. Cement Concrete Res. 2004, 34, 1889-1892.

[11] Mao Q, Yu C, Ge K. J. Tsinghua Univ. (Sci Tech) 2005, 45, 1672-1675 (in Chinese).

[12] Liu H, Tan H, Gao Q, Wang X, Xu T. Fuel 2010, 89, 3352-3357.

[13] Li B, Duan Y, Liu S. Constr. Build. Mater. 2012, 27, 184-188.

[14] Duan Y, Yang Y, Ma H. J. Phys. D Appl. Phys, 2008, 41, 1812-1854.

[15] Duan YP, Wang L, Liu Z, Gu SC. Plast. Rubber Compos. 2013, 42, 82-87.

[16] Zhang X, Sun W. Cement Concrete Comp. 2010, 32, 726-730.

[17] Benezet JC, Adamiec P, Benhassaine A. Particuology 2008, 6, 85-92.

[18] Chen L, Duan Y, Liu L, Guo J, Liu S. Mater. Design 2011, 32, 570-574.

[19] Michielssen E, Sager JM, Ranjithan S, Mittra R. Microwave Theory Tech. 1993, 41, 1024-1031.

[20] Duan YP, Liu SH, Wen B, Guan HT, Wang GQ. J. Compos. Mater. 2006, 40, 1841.

[21] Biwa S, Idekoba S, Ohno N. Mech. Mater. 2002, 34, 671-682.

[22] Matitsine SM, Hock KM, Liu L, Gan YB, Lagarkov AN, Rozanov KN. J. Appl. Phys. 2003, 94, 1146.

[23] Febrillet N, Akihiro K, Yukihiro I, Kouji I. Trans. Jpn. Concr. Inst. 2000, 22, 243-252.

[24] Huang CH, Lin SK, Chang CS, Chen HJ. Constr. Build. Mater. 2013, 46, 71-78. 